\title{
Stabilization of Néel order in frustrated magnets with increasing magnetic field
}

\author{
Burkhard Schmidt ${ }^{\mathrm{a}}$, Mohammad Siahatgar, and Peter Thalmeier \\ Max-Planck-Institut für Chemische Physik fester Stoffe, Dresden, Germany
}

\begin{abstract}
For low-dimensional frustrated quantum magnets, the dependence of the staggered moment $m_{\mathrm{s}}$ on a magnetic field is nonmonotonic: For small and intermediate fields, quantum fluctuations are gradually suppressed, leading to an increase of $m_{\mathrm{s}}(H)$. For large applied magnetic fields however, the classically expected monotonous decrease is recovered. For the same reasons, the Néel ordering temperature $T_{\mathrm{N}}$ of such compounds first increases and then exhibits a reentrant behavior as a function of the field strength. The quantitative analysis of this behavior is an excellent tool to determine the frustration parameter of a given compound. We have derived a general linear spin-wave (LSW) theory in the presence of a magnetic field. Based on our LSW theory, including a small interlayer coupling, we use a self-consistent approach determining $T_{\mathrm{N}}$ by the condition of a vanishing total moment. We apply our findings to the recently measured field dependence of the magnetic ordering temperature $T_{\mathrm{N}}$ of $\mathrm{Cu}(\mathrm{pz})_{2}\left(\mathrm{ClO}_{4}\right)_{2}$ in the framework of the $S=1 / 2$ two-dimensional $J_{1}-J_{2}$ Heisenberg model. The observed increase with increasing field strength can be understood naturally using an intermediate frustration ratio $J_{2} / J_{1} \approx$ 0.2 , which is in accordance with the field dependence of the staggered moment.
\end{abstract}

\section{Introduction}

The two-dimensional $J_{1}-J_{2}$ Heisenberg model on a square lattice with $S=1 / 2$ provides an excellent means for studying the interplay between quantum fluctuations already present in the nonfrustrated $J_{2}=0$ case, frustration effects in the magnetic exchange, and the impact of an applied magnetic field $H$. It has experimental realizations in two classes of layered $\mathrm{V}^{4+}$ compounds $[1,2]$ with an effective pseudospin $S=1 / 2$. An orthorhombically distorted version of the model can describe the magnetic structure and spin wave excitations of the undoped $122 \mathrm{Fe}$-pnictide compounds [3].

Another layered material with spin $S=1 / 2\left(\mathrm{Cu}^{2+}\right.$ $3 d^{9}$ configurations) is deuterated copper pyrazine perchlorate, $\mathrm{Cu}(\mathrm{pz})_{2}\left(\mathrm{ClO}_{4}\right)_{2}$. It crystallizes in a monoclinic crystal structure with space group $C 2 / c$. The $\mathrm{Cu}^{2+}$ ions form perfect square lattice layers lying in the crystallographic $b c$ plane with superexchange paths mediated by the pyrazine ligand molecules. Recent neutron scattering and thermodynamic experiments revealed an unusual field dependence of the magnetic Bragg scattering intensity and the transition temperature $T_{\mathrm{N}}$ to a Néel ordered state, both being stabilized by the magnetic field [4]. We will show that the observed field dependence can be naturally understood in the framework of the $J_{1}-J_{2}$ model.

In this paper, we emphasize the impact of the fieldinduced suppression of quantum fluctuations at finite temperatures on the anomalous enhancement of the Néel temperature. To describe this within the framework of our model, we introduce a small but finite interlayer coupling $J^{\prime}$ and determine $T_{\mathrm{N}}$ via the condition of vanishing expectation value of the total moment $m_{\mathrm{t}}$ defined below.

The properties of this model with nearest-neighbor exchange $J_{1}$ and next-nearest neighbor exchange $J_{2}$ (see be-

\footnotetext{
a e-mail: bs@cpfs.mpg.de
}

low for the definition of the Hamiltonian) are entirely determined by the frustration angle $\phi=\tan ^{-1}\left(J_{2} / J_{1}\right)$ with $-\pi<\phi \leq \pi$. In zero field, classically three phases exist: For $\tan ^{-1}(1 / 2) \leq \phi \leq \pi-\tan ^{-1}(1 / 2)(0.15 \lesssim \phi / \pi \lesssim 0.85)$, the ground state has columnar magnetic order with ordering vector $\mathbf{Q}=(\pi, 0)$ or $(0, \pi)$. For $-\pi / 2 \leq \phi \leq \tan ^{-1}(1 / 2)$, the ground state is Néel ordered with $\mathbf{Q}=(\pi, \pi)$, and for $-\pi<\phi \leq-\pi / 2$ and $\pi-\tan ^{-1}(1 / 2) \leq \phi \leq \pi$, the ground state is ferromagnetic. Quantum fluctuations destroy the magnetic order at the edges of the columnar phase: Around $\phi / \pi \approx 0.15\left(J_{2} / J_{1} \approx 1 / 2\right)$, a nonmagnetic stacked-dimer phase appears [5], and around $\phi / \pi \approx 0.85\left(J_{2} / J_{1} \approx-1 / 2\right)$, the ground state has spin-nematic character [6].

Therefore, a precise experimental determination of the frustration angle $\phi$ is of great importance in understanding the characteristics of a low-dimensional exchange-frustrated magnet. Several tools have already been developed: Measurements of the heat capacity and the magnetic susceptibility as a function of temperature and magnetic field can be discussed with the finite-temperature Lanczos method [7] or compared to a high-temperature series expansion [8]. With these methods we get the overall energy scale $J_{\mathrm{c}}=\sqrt{J_{1}^{2}+J_{2}^{2}}$ and the Weiss temperature $\Theta=J_{1}+$ $J_{2}$. An ambiguity remains as the determination of $\Theta$ and $J_{\mathrm{c}}$ alone does not specify $J_{1}$ and $J_{2}$ completely. An analysis of the saturation field can also help determining $\phi$ or $J_{2} / J_{1}$ [9]. However, saturation fields frequently are too large to be experimentally accessible.

Here we discuss an independent method for the precise determination of the frustration angle $\phi$ which can in principal be applied to any low-dimensional frustrated magnet with a magnetically ordered ground state. This method has the advantage that only the initial slope of the field dependence of the ordered moment or Néel temperature is required to determine the frustration ratio, making it ap-

This is an Open Access article distributed under the terms of the Creative Commons Attribution License 2.0, which permits unrestricted use, distribution, and reproduction in any medium, provided the original work is properly cited. Article available at nttp://www.epj-conterences.org or nttp://dx.dol.org/10.1051/epjcont/20134004001 
plicable to a much larger range of compounds with comparatively high saturation fields. As was shown in [10,11], quantum effects lead to a nonlinear uniform magnetization. The quantum fluctuations affect the staggered moment even more strongly $[12,13]$. We will give an overview over the linear spin-wave (LSW) theory applied to our model. For $\mathrm{Cu}(\mathrm{pz})_{2}\left(\mathrm{ClO}_{4}\right)_{2}$, we restrict to the Néel phase. The unusual field dependence of the Néel ordering temperature can be understood within a self-consistent treatment of the total moment $m_{\mathrm{t}}$ at finite temperatures within spin-wave theory in the spirit of an RPA approach $[14,15]$.

\section{Frustrated Heisenberg model}

The Hamiltonian we use is given by

$$
\begin{aligned}
H= & J_{1} \sum_{\langle i, j\rangle_{1}} \mathbf{S}_{i} \mathbf{S}_{j}+J_{2} \sum_{\langle i, j\rangle_{2}} \mathbf{S}_{i} \mathbf{S}_{j} \\
& +J^{\prime} \sum_{\langle i, j\rangle_{\perp}} \mathbf{S}_{i} \mathbf{S}_{j}-g \mu_{\mathrm{B}} \mathbf{H} \sum_{i} \mathbf{S}_{i} .
\end{aligned}
$$

The first sum runs over bonds connecting nearest-neighbor sites $i$ and $j$, the second sum runs over bonds connecting next-nearest neighbors in the adjacent $\mathrm{Cu}$ planes. The third sum parametrizes the exchange coupling between adjacent layers with $J^{\prime} / J_{\mathrm{c}} \ll 1$. The last term is the Zeeman energy in an applied field $\mathbf{H}$ which we choose to be parallel to the $x$ direction. As usual, $g$ denotes the gyromagnetic ratio, and $\mu_{\mathrm{B}}$ is the Bohr magneton. Exchange anisotropies are not included. The two major exchange constants are $J_{1}=$ $J_{\mathrm{c}} \cos \phi$ and $J_{2}=J_{\mathrm{c}} \sin \phi$.

We can express the field-dependent total moment in units of $g \mu_{\mathrm{B}}$ with its components parallel $\left(m_{0}\right)$ and perpendicular $\left(m_{\mathbf{Q}}\right)$ to the direction of the applied field,

$$
m_{\mathrm{t}}^{2}(h)=m_{0}^{2}(h)+m_{\mathbf{Q}}^{2}(h)
$$

with $h=g \mu_{\mathrm{B}} H$.

\section{Classical ground state}

We have described the calculation of uniform and staggered moment within linear spin-wave theory to great detail in Ref. [3]. Here we restrict ourselves to giving an overview, including the interlayer coupling $J^{\prime}$ into the exchange model. We assume the magnetic field to be aligned parallel to the crystallographic $a$ axis of $\mathrm{Cu}$ pyrazine. Classically the uniform and staggered moments are given by the respective projections of the ordered spins onto the field axis:

$$
\begin{aligned}
& m_{0}=S \cos \Theta_{\mathrm{c}}, \\
& m_{\mathbf{Q}}=S \sin \Theta_{\mathrm{c}},
\end{aligned}
$$

where $\Theta_{c}$ is the classical canting angle given by

$$
\cos \Theta_{\mathrm{c}}=h / h_{\mathrm{sat}}
$$

with a saturation field $h_{\text {sat }}=g \mu_{\mathrm{B}} H_{\text {sat }}=4 J_{1}$ in the Néel phase with an in-plane ordering vector $\mathbf{Q}=(\pi, \pi)$. That is, the size of the total moment $m_{\mathrm{t}}=S$ is field-independent, and the single effect of the increasing magnetic field is to tilt the spins out of the $b c$ plane towards the $a$ direction until at $h=h_{\text {sat }}$, full polarization with $m_{0}=S$ is reached. Note that the ordering vector has in principle three components. However the interlayer interaction $J^{\prime}$ along the $a$ direction is small [4], thus the precise ordering along this direction does not play a role in our discussion.

\section{Linear spin-wave theory}

Quantum fluctuations in the ground state modify this simple classical picture of moments as tilting vectors: Already at field $h=0$ and next-nearest neighbor coupling $J_{2}=0$, the expectation value of the staggered moment or "length" of the moment is reduced from $m_{\mathbf{Q}}=1 / 2$ to $m_{\mathbf{Q}} \approx 0.3$. A finite antiferromagnetic value of $J_{2}$ reduces $m_{\mathbf{Q}}$ even further [3]. In addition to the canting angle, this length now is a function of the field $h$, too, because its classical value at $h=h_{\text {sat }}$ being represented by the uniform moment $m_{0}\left(h_{\text {sat }}\right)=S$ must be recovered. This means, the field dependence of $m_{0}$ and $m_{\mathbf{Q}}$ is governed by both the length and the canting angle of the total moment $m_{\mathrm{t}}$, and all of them are controlled by the single frustration parameter $\phi$.

On each site $i$, we introduce a local coordinate system where the $x$ axis is oriented parallel to the local magnetic moment. We apply a Holstein-Primakoff transformation to the spin operators expressed in this coordinate system,

$$
\begin{aligned}
& S_{i}^{x}=S-a_{i}^{\dagger} a_{i}, \\
& S_{i}^{+}=\sqrt{2 S}\left(1-\frac{a_{i}^{\dagger} a_{i}}{2 S}\right)^{1 / 2} a_{i} \rightarrow \sqrt{2 S} a_{i}, \\
& S_{i}^{-}=\sqrt{2 S} a_{i}^{\dagger}\left(1-\frac{a_{i}^{\dagger} a_{i}}{2 S}\right)^{1 / 2} \rightarrow \sqrt{2 S} a_{i}^{\dagger} .
\end{aligned}
$$

The operators $a_{i}$ and $a_{i}^{\dagger}$ represent a boson field of spin excitations, and we keep only terms up to bilinear order in the Hamiltonian.

Next we insert a Fourier representation of the bosonic field,

$$
a_{i}^{\dagger}=\frac{1}{\sqrt{N}} \sum_{\mathbf{k}} a_{\mathbf{k}}^{\dagger} e^{-\mathbf{i} \mathbf{k} \mathbf{R}_{i}},
$$

into the resulting Hamiltonian and introduce magnon operators

$$
\alpha_{\mathbf{k}}=u_{\mathbf{k}} a_{\mathbf{k}}+v_{\mathbf{k}} a_{-\mathbf{k}}^{\dagger}, \quad \alpha_{-\mathbf{k}}^{\dagger}=v_{\mathbf{k}} a_{\mathbf{k}}+u_{\mathbf{k}} a_{-\mathbf{k}}^{\dagger} \cdot
$$

Writing Eq. (1) with these operators and requiring the resulting Hamiltonian to have diagonal form diagonalizes the Hamiltonian in the usual way.

The total moment $m_{\mathrm{t}} \equiv\langle S\rangle$ is given by the expectation value of the $x$ component of the spin $\mathbf{S}$ in local coordinates,

$$
\begin{aligned}
\langle S\rangle & =\frac{1}{N} \sum_{i}\left\langle S_{i}^{x}\right\rangle=\frac{1}{N} \sum_{i}\left\langle S-a_{i}^{\dagger} a_{i}\right\rangle \\
& =S-\frac{1}{N} \sum_{\mathbf{k}}\left\langle a_{\mathbf{k}}^{\dagger} a_{\mathbf{k}}\right\rangle,
\end{aligned}
$$

where the brackets $\langle\ldots\rangle$ in the last equation denote the trace over the statistical operator. The operator $a_{\mathbf{k}}^{\dagger} a_{\mathbf{k}}$ is not diagonal in the basis spanned by the magnons $\alpha_{\mathbf{k}}^{\dagger}$, but rather 
is related by the Bogoliubov transformation $U$ (see Eq. (10)) with $\operatorname{det}(U)=1$ given by the matrix in

$$
\left(\begin{array}{c}
\alpha_{\mathbf{k}} \\
\alpha_{-\mathbf{k}}^{\dagger}
\end{array}\right)=\left(\begin{array}{cc}
u_{\mathbf{k}} & v_{\mathbf{k}} \\
v_{\mathbf{k}} & u_{\mathbf{k}}
\end{array}\right)\left(\begin{array}{c}
a_{\mathbf{k}} \\
a_{-\mathbf{k}}^{\dagger}
\end{array}\right)
$$

With

$$
\left\langle\alpha_{-\mathbf{k}} \alpha_{-\mathbf{k}}^{\dagger}\right\rangle=1+\left\langle\alpha_{-\mathbf{k}}^{\dagger} \alpha_{-\mathbf{k}}\right\rangle=1+n_{-k}
$$

we have

$$
\begin{aligned}
\left\langle a_{\mathbf{k}}^{\dagger} a_{\mathbf{k}}\right\rangle & =u_{\mathbf{k}}^{2} n_{\mathbf{k}}+v_{\mathbf{k}}^{2}\left(1+n_{-\mathbf{k}}\right) \\
& =\left(1+v_{\mathbf{k}}^{2}\right) n_{\mathbf{k}}+v_{\mathbf{k}}^{2}\left(1+n_{-\mathbf{k}}\right) \\
& \stackrel{T \rightarrow 0}{\longrightarrow} v_{\mathbf{k}}^{2} .
\end{aligned}
$$

With the standard Bose occupation numbers $n_{\mathbf{k}}=1 /\left(\mathrm{e}^{\beta \omega_{\mathbf{k}}}-\right.$ $1), \beta \equiv 1 /\left(k_{\mathrm{B}} T\right)$, the magnon excitation energies $\omega_{\mathbf{k}}=$ $S E(h, \mathbf{k})$ and using $n_{\mathbf{k}}=n_{-\mathbf{k}}$, we eventually get

$$
\left\langle a_{\mathbf{k}}^{\dagger} a_{\mathbf{k}}\right\rangle=v_{\mathbf{k}}^{2}+\left(1+2 v_{\mathbf{k}}^{2}\right) n_{\mathbf{k}} .
$$

The total moment at finite temperatures $T$ is thus given by $\langle S\rangle=S-\Psi$ with

$$
\Psi=\frac{1}{N} \sum_{\mathbf{k}}\left[v_{\mathbf{k}}^{2}+\left(1+2 v_{\mathbf{k}}^{2}\right) n_{\mathbf{k}}\right] .
$$

Explicit diagonalization of the Hamiltonian (1) yields

$$
v_{\mathbf{k}}^{2}=\frac{1}{2}\left(\frac{A(\mathbf{k})-B(\mathbf{k}) \cos ^{2} \Theta_{\mathrm{c}}}{E(h, \mathbf{k})}-1\right) .
$$

The spin-wave energies are given by

$$
\begin{aligned}
E_{\mathbf{k}}(h)= & \left\{\left[A_{\mathbf{k}}-B_{\mathbf{k}} \cos ^{2} \Theta_{\mathrm{c}}\right]^{2}\right. \\
& \left.-\left[B_{\mathbf{k}}\left(1-\cos ^{2} \Theta_{\mathrm{c}}\right)\right]^{2}\right\}^{\frac{1}{2}}, \\
A_{\mathbf{k}}= & J_{\mathbf{k}}+\frac{1}{2}\left(J_{\mathbf{k}+\mathbf{Q}}+J_{\mathbf{k}-\mathbf{Q}}\right)-2 J_{\mathbf{Q}}, \\
B_{\mathbf{k}}= & J_{\mathbf{k}}-\frac{1}{2}\left(J_{\mathbf{k}+\mathbf{Q}}+J_{\mathbf{k}-\mathbf{Q}}\right),
\end{aligned}
$$

and $J_{\mathbf{k}}=2 J^{\prime} \cos k_{x}+2 J_{1}\left(\cos k_{y}+\cos k_{z}\right)+4 J_{2} \cos k_{y} \cos k_{z}$ is the Fourier transform of the exchange couplings.

\section{Field dependent transition temperature}

The Néel order at finite $T_{\mathrm{N}}$ is an effect of the finite interlayer coupling $J^{\prime}$. Because of the quasi-long range order of the two-dimensional Heisenberg antiferromagnet with exponentially increasing correlation length at small temperatures even a small interlayer coupling $J^{\prime} / J_{\mathrm{c}} \ll 1$ leads to sizable $T_{\mathrm{N}}$ on the scale of the intra-layer exchange strength [16]. $T_{\mathrm{N}}(0)$ for the nonfrustrated model $(\phi=0)$ may be obtained from an empirical formula based on Monte Carlo simulations, however, this is not available for $\phi \neq 0$ and for finite fields.

At the temperature $T_{\mathrm{N}}$ where magnetic order breaks down, the total moment vanishes. This condition can be written as

$$
\langle S\rangle=S-\Psi=0
$$

for the expectation value of the spin in the local coordinate system with $\Psi$ given by Eq. (17) . Compared to a selfconsistent RPA theory $[14,15]$ based on quasi-two-dimensional spin waves where $T_{\mathrm{N}}(h)$ is determined by the condition of vanishing staggered moment, for $S=1 / 2$ this equation can be formally regarded as the first two terms of a small- $\Psi$ series expansion of the self-consistent equation

$$
\langle S\rangle=\frac{1 / 2}{1+2 \Psi} .
$$

Here inside $\Psi$ the classical value $S$ occurring in $\omega_{\mathbf{k}}=$ $S E(h, \mathbf{k})$ has to be replaced with the expectation value $\langle S\rangle$, which is then to be determined self-consistently. Having done this replacement in the spirit of RPA, we can assume that $\langle S\rangle \ll S$ near $T=T_{\mathrm{N}}$, and the argument of the Bose function is small, thus we can further approximate

$$
\begin{aligned}
\frac{1}{\mathrm{e}^{\beta \omega_{\mathbf{k}}}-1} & \approx \frac{1}{\beta \omega_{\mathbf{k}}}-\frac{1}{2} \\
\Psi & \approx \frac{1}{N} \sum_{\mathbf{k}}\left[v_{\mathbf{k}}^{2}+\left(1+2 v_{\mathbf{k}}^{2}\right)\left(\frac{1}{\beta \omega_{\mathbf{k}}}-\frac{1}{2}\right)\right] \\
& =\frac{k_{\mathrm{B}} T}{\langle S\rangle} \frac{1}{N} \sum_{\mathbf{k}} \frac{1+2 v_{\mathbf{k}}^{2}}{E(h, \mathbf{k})}-\frac{1}{2}
\end{aligned}
$$

Inserting this expression for $\Psi$ into Eq. (23) yields

$$
\begin{aligned}
\langle S\rangle & =\frac{1}{2}\left(1+2 \frac{k_{\mathrm{B}} T}{\langle S\rangle} \frac{1}{N} \sum_{\mathbf{k}} \frac{1+2 v_{\mathbf{k}}^{2}}{E(h, \mathbf{k})}-1\right)^{-1} \\
& =\left(4 \frac{k_{\mathrm{B}} T}{\langle S\rangle} \frac{1}{N} \sum_{\mathbf{k}} \frac{1+2 v_{\mathbf{k}}^{2}}{E(h, \mathbf{k})}\right)^{-1}
\end{aligned}
$$

where now $\langle S\rangle$ drops out and we eventually get

$$
T_{\mathrm{N}}(h)=\left(4 k_{\mathrm{B}} \frac{1}{N} \sum_{\mathbf{k}} \frac{A(\mathbf{k})-B(\mathbf{k}) \cos ^{2} \Theta_{\mathrm{c}}}{E^{2}(h, \mathbf{k})}\right)^{-1}
$$

for the field dependence of the Néel transition temperature within our approximations.

\section{Application to $\mathrm{Cu}(\mathrm{pz})_{2}\left(\mathrm{ClO}_{4}\right)_{2}$}

In Ref. [4], the $H-T$ phase diagram of $\mathrm{Cu}(\mathrm{pz})_{2}\left(\mathrm{ClO}_{4}\right)_{2}$ has been determined experimentally from the neutron scattering intensity at a particular antiferromagnetic Bragg peak. Heat capacity measurements in magnetic field applied parallel and perpendicular to the $\mathrm{Cu}$ square-lattice planes have been performed as well. These results are displayed in Fig. 1. Open circles label the peak positions of the temperature dependence of the heat capacity, while triangles denote the neutron scattering results.

Eq. (27) reduces to the expression in Ref. [14] for $h=$ 0 . The theory predicts the right dependence of $T_{\mathrm{N}}(0)$ on $J^{\prime} / J_{1}$ but the absolute values are larger than those derived from empirical formulas fitting the Monte Carlo simulations (at $J_{2}=0$ ) [16]. Therefore in Fig. 1 we have plotted the field dependent transition temperature $T_{\mathrm{N}}(h) / T_{\mathrm{N}}(0)$ normalized to its value at $h=0$. It is shown for two sets of values $\left(\phi, J^{\prime}\right)$. The main effect of the increasing field is the reduction of quantum fluctuations. This in turn increases 


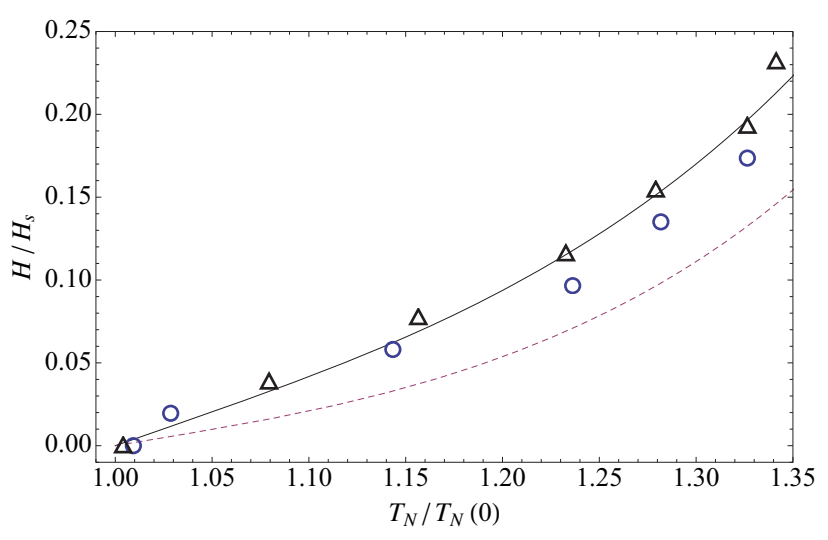

Fig. 1. HT phase diagram of $\mathrm{Cu}(\mathrm{pz})_{2}\left(\mathrm{ClO}_{4}\right)_{2}$. The magnetic transition temperature $T_{\mathrm{N}}$ is normalized to $T_{\mathrm{N}}(H=0)$, the applied magnetic field $H$ is normalized to the saturation field $H_{\text {sat }}$. The symbols label experimental values determined from heat capacity measurements (open circles) and neutron diffraction (triangles) [4]. The lines show the results from our RPA spin-wave calculations with $\phi=0.063 \pi\left(J_{2} / J_{1}=0.2\right), J^{\prime} / J_{1}=0.006$ (solid line) and $\phi=0.006 \pi\left(J_{2} / J_{1}=0.02\right), J^{\prime} / J_{1}=6.8 \times 10^{-4}$ (dashed line).

the spin-wave energy $E(h, \mathbf{k})$ in the argument of the integral in Eq. (27), leading to the experimentally observed enhancement of $T_{\mathrm{N}}(h)$.

The $\left(\phi, J^{\prime}\right)$ values for the dashed curve, corresponding to a small frustration ratio $J_{2} / J_{1} \approx 0.018$ in Fig. 1 reproduce the experimental $T_{\mathrm{N}}(0)=4.2 \mathrm{~K}$ but fail for the field dependence. Taking the frustration ratio $J_{2} / J_{1}=0.2$ or equivalently $\phi=0.063 \pi$ determined from the field dependence of the ordered moment [13], excellent agreement with the experimental $T_{\mathrm{N}}(h) / T_{\mathrm{N}}(0)$ is achieved with $J^{\prime} / J_{1}=$ 0.006 . However, the transition temperature $T_{\mathrm{N}}(0)$ is about twice the experimental value in this case. We think that the functional dependence $T_{\mathrm{N}}(h)$ is more significant for $J^{\prime} / J_{1}$ than the single value of $T_{\mathrm{N}}(0)$.

In Ref. [4], also the low-temperature peak intensity of neutron scattering at an antiferromagnetic point has been measured as a function of the applied magnetic field. Apart from a constant, this intensity is proportional to the static magnetic structure factor at the antiferromagnetic ordering vector, identical to the staggered moment $m_{\mathbf{Q}}^{2}$. Within the framework of our LSW theory, we have fitted $m_{\mathbf{Q}}^{2}$ to the neutron data [13]. The best agreement is obtained with $J_{2} / J_{1} \approx 0.2$ which is also the value which is used in Fig. 1. This clearly indicates that $\mathrm{Cu}$ pyrazine is a moderately frustrated Néel antiferromagnet .

\section{Conclusion}

The anomalous field dependence of the Néel transition temperature $T_{\mathrm{N}}$ of the two-dimensional frustrated $J_{1}-J_{2}$ Heisenberg model with $S=1 / 2$ on a square lattice has been discussed. A single parameter $\phi=\tan ^{-1}\left(J_{2} / J_{1}\right)$ controls the properties of this model. With an additional small interlayer coupling $J^{\prime}$, we have used linear spin-wave theory supplemented by a selfconsistency condition in the spirit of RPA theory to derive $T_{\mathrm{N}}(h)$. A finite frustration ratio $J_{2} / J_{1}$ results in an increase of quantum effects, and the field-induced suppression of quantum fluctuations leads to the anomalous increase of $T_{\mathrm{N}}(h)$ observed experimentally in $\mathrm{Cu}(\mathrm{pz})_{2}\left(\mathrm{ClO}_{4}\right)_{2}$. With $J_{2} / J_{1} \approx 0.2$, we have achieved an excellent agreement of $T_{\mathrm{N}}(h) / T_{\mathrm{N}}(0)$ with the experimental $H-T$ phase diagram.

\section{References}

1. R. Melzi, P. Carretta, A. Lascialfari, M. Mambrini, M. Troyer, P. Millet, F. Mila, Phys. Rev. Lett. 85, 1318 (2000)

2. N.S. Kini, E.E. Kaul, C. Geibel, J. Phys.: Cond. Mat. 18, 1303 (2006)

3. B. Schmidt, M. Siahatgar, P. Thalmeier, Phys. Rev. B 81, 165101 (2010)

4. N. Tsyrulin, F. Xiao, A. Schneidewind, P. Link, H.M. Rønnow, J. Gavilano, C.P. Landee, M.M. Turnbull, M. Kenzelmann, Phys. Rev. B 81, 134409 (2010)

5. R.R.P. Singh, W. Zheng, C.J. Hamer, J. Oitmaa, Phys. Rev. B 60, 7278 (1999)

6. N. Shannon, T. Momoi, P. Sindzingre, Phys. Rev. Lett. 96, 027213 (2006)

7. N. Shannon, B. Schmidt, K. Penc, P. Thalmeier, Eur. Phys. J. B 38, 599 (2004)

8. G. Misguich, B. Bernu, L. Pierre, Phys. Rev. B 68, 113409 (2003)

9. A.A. Tsirlin, B. Schmidt, Y. Skourski, R. Nath, C. Geibel, H. Rosner, Phys. Rev. B 80, 132407 (2009)

10. P. Thalmeier, M.E. Zhitomirsky, B. Schmidt, N. Shannon, Phys. Rev. B 77, 104441 (2008)

11. B. Schmidt, M. Siahatgar, P. Thalmeier, Phys. Rev. B 83, 075123 (2011)

12. A. Lüscher, A.M. Läuchli, Phys. Rev. B 79, 195102 (2009)

13. M. Siahatgar, B. Schmidt, P. Thalmeier, Phys. Rev. B 84, 064431 (2011)

14. N. Majlis, S. Selzer, G.C. Strinati, Phys. Rev. B 45, 7872 (1992)

15. N. Majlis, S. Selzer, G.C. Strinati, Phys. Rev. B 48, 957 (1993)

16. C. Yasuda, S. Todo, K. Hukushima, F. Alet, M. Keller, M. Troyer, H. Takayama, Phys. Rev. Lett. 94, 217201 (2005) 